\title{
OPTIMAL CONTROL OF A CLASS OF STRONGLY NONLINEAR EVOLUTION SYSTEMS *
}

\author{
Z. H. Zhang \& X. Xiang \\ Department of Mathematics \\ Guizhou University \\ Guiyang, Guizhou, P.R. China.
}

\begin{abstract}
In this paper, we investigate the existence of optimal controls of the first-order nonlinear evolution systems whose principle operator is pseudomonotone operator with nonmonotone nonlinearities perturbation.
\end{abstract}

Keywords. Nonmonotone nonlinearities, Pseudomonotone operator, Optimality.

\section{Introduction}

Recently, optimal control problems of nonlinear distributed parameter systems have been extensively studied. Many authors pay great attention to several class of semilinear and nonlinear evolution equations, such as, Papageorgiou ([1]-[2]), Ahmed ([4]) and Xiang ([5]). Meanwhile, to our knowlege, the class of nonlinear control systems with pseudomonotone operator has rarely been studied.

As well-known, in studying existence of solutions of nonlinear evolution equations, we usually consider the evolution triples ([3]), $X \hookrightarrow H \hookrightarrow X^{*}$, or more generally, consider the chairs of spaces ([1]),

$$
X \hookrightarrow Y \hookrightarrow H \hookrightarrow Y^{*} \hookrightarrow X^{*} .
$$

The nonlinear perturbation is assumed by some authors as following:

$f: I \times Y \rightarrow Y^{*}([1])$, here $I$ denotes the time horizon, or $f: I \times H \rightarrow H([2])$, or $f: I \times H \rightarrow X^{*}([5])$. In this paper, by developing the concept of pseudomonotone operator and using some technique of convergence, we deal with the existence of solutions of nonlinear evolution equation whose principle operator is pseudomonotone operator with nonmonotone nonlinearities perturbation being from $I \times Y$ into $X^{*}$, which includes the results of [1] and [2]. Further,

*Projects Supported by Chinese National Natural Science Foundation 29362003 .

The original version of this chapter was revised: The copyright line was incorrect. This has been corrected. The Erratum to this chapter is available at DOI: 10.1007/978-0-387-35359-3_40 
we solve the existence of optimal controls of nonlinear control system.

\section{Preliminaries}

Let $T$ be a fixed number, and $I=[0, T]$ the time horizon, $H$ a separable Hilbert space, and $X, Y$ subspaces of $H$ carrying the structure of a separable reflexive Banach space. We will assume that $X \hookrightarrow Y \hookrightarrow H$, with all embeddings being continuous, dense, and $X$ into $Y$ is also compact. Identifying $H$ with its dual, we have

$$
X \hookrightarrow Y \hookrightarrow H \hookrightarrow Y^{*} \hookrightarrow X^{*},
$$

with all embeddings being continuous, dense, and the first and the last are also compact. In the literature, triples like $\left(X, H, X^{*}\right)$ and $\left(Y, H, Y^{*}\right)$ are usually called evolution triples $([3])$. For simplicity, we denote by $<., .>$ and $(.,$. the duality brackets of the pair $\left(X, X^{*}\right)$ and the inner product of $H$, respectively. They are compatible in the sense that $\left.\langle.,\rangle\right|_{.X \times H}=(.,$.$) (proposition$ $23.13,[3])$. Also, by $\|$.$\| (resp., \|.\|_{Y},|\cdot|,\|.\|_{Y^{*}},\|.\|_{*}$ ), we denote the norm of $X$ (resp., Y, H, $\left.Y^{*}, X^{*}\right)$, and by $\longrightarrow^{s}\left(\longrightarrow^{w}\right)$, we denote the strongly (weakly) convergence. We set

$$
W_{p q}=\left\{x \in L^{p}(I, X), \dot{x} \in L^{q}\left(I, X^{*}\right)\right\}\left(1<p, q<\infty, \frac{1}{p}+\frac{1}{q}=1\right),
$$

where the derivative in the definition should be understood in the sense of vector-valued distributions, equiped with the norm

$$
\|x\|_{W_{p q}}=\left(\|x\|_{L^{p}(I, X)}^{2}+\|\dot{x}\|_{L^{q}\left(I, X^{*}\right)}^{2}\right)^{\frac{1}{2}} .
$$

The space $W_{p q}$ becomes a separable reflexsive Banach space. Moreover, $W_{p q} \hookrightarrow L^{p}(I, X)$ and $W_{p q} \hookrightarrow C(I, H)$ continuously, and $W_{p q} \hookrightarrow L^{p}(I, Y)$ compactly (pp. 650, [1]). For convenience, we write the spaces

$V=L^{p}(I, X), V^{*}=L^{q}\left(I, X^{*}\right), \mathcal{H}=L^{2}(I, H), Z=L^{p}(I, Y), Z^{*}=$ $L^{q}\left(I, Y^{*}\right)$.

By $\|.\|_{V}$ (resp., \|\|$_{V^{*}},\|.\|_{\mathcal{H}},\|.\|_{Z},\|.\|_{Z^{*}}$ ), we denote the norm of the space $V$ (resp., $V^{*}, \mathcal{H}, Z, Z^{*}$ ). Let $<<.,>>$ and $((.,)$.$) denote the duality brackets$ for the pair $\left(V, V^{*}\right)$ and the inner product of $\mathcal{H}$, respectively.

Next we model the control space by a reflexive separable B-space $E$ with the norm $\|.\|_{E}$. By $P_{f(c)}(E)$, we denote a class of nonempty, closed (convex) subsets of $E$. Recall that a multi-function $U: I \rightarrow P_{f}(E)$ is called measurable if

$$
G r U=\{(t, v) \in I \times E: v \in U(t)\} \in B(I) \times B(E),
$$

with $B(I)(B(E))$ being the Borel $\sigma$-field of $I(E)$. By $\mathcal{U}_{a d}$, we denote the admissible control set of all selectors of $U($.$) that belong to Lebesgue-Bochner$ space $L^{q}(I, E), 1<q<\infty$; that is,

Note that

$$
\mathcal{U}_{a d}=\left\{u \in L^{q}(I, E): u(t) \in U(t) \text {, a.e. on } I\right\} .
$$

$$
\mathcal{U}_{a d} \neq \phi, \text { if } t \rightarrow|U(t)|=\sup \left\{\|u\|_{E}, u \in U(t)\right\} \in L_{+}^{q},
$$

in which case the multi-function is said to be $L^{q}$-bounded.

Finally recall that an operator $A: X \rightarrow X^{*}$ is said to be pseudomonotone if, for each $x \in X$ and each sequence $\left(x_{n}\right)$ in $\mathrm{X}$,

$$
x_{n} \longrightarrow^{w} x \text { in } \mathrm{X} \text { as } n \rightarrow \infty \text { and } \varlimsup_{n \rightarrow \infty}<A x_{n}, x_{n}-x>\leq 0
$$


imply

$$
<A x, x-w>\leq \underline{\lim }_{n \rightarrow \infty}<A x_{n}, x_{n}-w>\text {, for all } w \in X .
$$

In order to verify the existence of solutions of System (3.1)(See next section), we develop the concept of pseudomonotone.

Definition An operator $A: M \subseteq X \rightarrow X^{*}$ on the complete subspace $M$ of the real Banach space $X$ is called quasi-pseudomonotone if, for each $x \in M$ and each sequence $\left(x_{n}\right)$ in $M$,

$$
x_{n} \longrightarrow^{w} x \text { in } M \text { as } n \rightarrow \infty \text { and } \varlimsup_{n \rightarrow \infty}<A x_{n}, x_{n}-x>\leq 0,
$$

there exists a subsequence $\left(x_{n_{k}}\right)$ of $\left(x_{n}\right)$, s.t.

$$
<A x, x-w>\leq \underline{\lim }_{k \rightarrow \infty}<A x_{n_{k}}, x_{n_{k}}-w>\text {, for all } w \in X \text {. }
$$

\section{Existence of Solutions of Nonlinear Evolution Equation}

We consider the following initial value problem:

$$
\left\{\begin{array}{l}
\dot{x}(t)+A(t, x(t))=f(t, x(t)), \quad \text { a.e.onI } \\
x(0)=x_{0}
\end{array}\right.
$$

Recall that a function $x \in W_{p q}$ is a solution of (3.1) if, for all $v \in X, x$ satisfies

$$
\left\{\begin{array}{l}
\frac{d}{d t}(x(t), v)+<A(t, x(t)), v>=<f(t, x(t)), v>, \quad \text { a.e.onI } \\
x(0)=x_{0}
\end{array}\right.
$$

where $\dot{x}$ means the generalized distributed derivatives.

In order to investigate the existence of solutions of $(3.1)$, we need the following hypotheses.

Hypothesis (A). $A: I \times X \rightarrow X^{*}$ is an operator s.t.

(a) $t \rightarrow A(t, x)$ is measurable;

(b) $\quad x \rightarrow A(t, x)$ is pseudomonotone;

(c) $\quad\|A(t, x)\|_{*} \leq a_{1}(t)+b_{1}\|x\|^{p-1}$,

for all $x \in X$, with $a_{1} \in L_{+}^{q}(0, T), b_{1}>0,2 \leq p<\infty, \frac{1}{p}+\frac{1}{q}=1$;

(d) $\quad<A(t, x)-A(t, y), x-y>\geq c\|x-y\|^{p}-d|x-y|^{2}$, for all $x, y \in X$, with $c>0$ and $d \geq 0$.

Hypothesis (F). $f: I \times Y \rightarrow \overline{X^{*}}$ is also an operator s.t.

(a) $\quad t \rightarrow f(t, x)$ is measurable;

(b) $\quad x \rightarrow f(t, x)$ is continuous from $\mathrm{Y}$ into $\mathrm{X}^{*}$;

(c) $\quad\|f(t, x)\|_{*} \leq a_{2}(t)+b_{2}\|x\|_{Y}^{p-1}$

for all $x \in Y$, with $a_{2} \in L_{+}^{q}(0, T)$ and $b_{2}>0$;

(d) $\quad\left\langle f(t, x), x>\leq \phi(t)\right.$, for all $x \in X$, with $\phi \in L^{1}(0, T)$.

Hypothesis $\left(\mathbf{H}_{0}\right) . x_{0} \in H$.

Next we define the Nemyckii operators $\widehat{A}$ and $\widehat{f}$, given by

$$
(\widehat{A} x)(t)=A(t, x(t)) \text {, for } x \in V ;(\widehat{f} x)(t)=f(t, x(t)) \text {, for } x \in Z \text {. }
$$

Let $\left.\widehat{A}\right|_{W_{p q}}$ denote the largest restriction of $\widehat{A}$ on $W_{p q}$. We have some important properties on the operators $\widehat{A}, \widehat{f}$ and $\left.\widehat{A}\right|_{W_{p q}}$.

Lemma 1. Under Hypothesis (A), $\widehat{A}: V \rightarrow V^{*}$ is bounded and satisfies 
(a) $\quad\|\widehat{A} x\|_{V} \cdot \leq \operatorname{const}\left(\left\|a_{1}\right\|_{q}+b_{1}\|x\|_{V}^{p-1}\right)$, for all $x \in V$;

(b) $\quad<<\widehat{A} x-\hat{A} y, x-y>>\geq c\|x-y\|_{V}^{p}-d\|x-y\|_{\mathcal{H}}^{2}$,

for all $x, y \in V$;

(c) (i) $\left.\widehat{A}\right|_{W_{p q}}$ is quasi-pseudomonotone from $W_{p q} \subseteq V$ into $V^{*}$.

(ii) $\left.\widehat{A}\right|_{W_{p q}}$ satisfies the condition(M), i.e.

$x_{n} \longrightarrow^{w} x$ in $W_{p q}, \widehat{A} x_{n} \longrightarrow^{w} b$ in $V^{*}$, and $\varlimsup_{n \rightarrow \infty}<<\widehat{A} x_{n}, x_{n}>>\leq$ $<<b, x>>$,

imply

$$
\widehat{A} x=b .
$$

Proof. One can easily verify that (a) and (b) hold true. Now we show (c)(i).

Let $x_{n} \longrightarrow^{w} x$ in $W_{p q}$ and $\overline{\lim }_{n \rightarrow \infty}<<\widehat{A} x_{n}, x_{n}-x>>\leq 0$.

Thanks to (b), we have

$$
\begin{aligned}
& c \varlimsup_{\lim _{n \rightarrow \infty}}\left\|x_{n}-x\right\|_{V}^{p} \leq d \varlimsup_{\lim _{n \rightarrow \infty}}\left\|x_{n}-x\right\|_{\mathcal{H}}^{2} \\
& +\varlimsup_{n \rightarrow \infty}<<\widehat{A} x_{n}, x_{n}-x>> \\
& -\lim _{n \rightarrow \infty}<<\widehat{A} x, x_{n}-x>>\leq 0 .
\end{aligned}
$$

Since $W_{p q} \hookrightarrow \mathcal{H}=L^{2}(I, H)$ compactly, we get that $x_{n} \longrightarrow^{s} x$ in $\mathcal{H}$. Hence, it follows that $x_{n} \longrightarrow^{s} x$ in $V=L^{p}(I, X)$. Thus, there exists a subsequence of $\left(x_{n}\right)$, denoted by $\left(x_{n_{k}}\right)$, such that $x_{n_{k}}(t) \longrightarrow^{s} x(t)$ in $X$, a.e.on $I$, and there exists a real function $v \in L^{p}(0, T)$ satisfying

(See pp. 579, [3]).

$$
\left\|x_{n_{k}}(t)\right\| \leq v(t), \text { for a.e. } t \in I
$$

By virtue of Hypothesis (A)(c), we can show that

$$
\lim _{k \rightarrow \infty}<A\left(t, x_{n_{k}}(t)\right), x_{n_{k}}(t)-x(t)>=0 \text {, a.e. on } I \text {. }
$$

Since $A$ is pseudomonotone, we have

$$
<A(t, x(t)), x(t)-w(t)>\leq \underline{\lim }_{k \rightarrow \infty}<A\left(t, x_{n_{k}}(t)\right), x_{n_{k}}(t)-w(t)>d t,
$$

a.e. on $I$, for all $w \in V$. And consequently,

$$
<\widehat{A} x, x-w>>\leq \int_{I} \varliminf_{k \rightarrow \infty}<A\left(t, x_{n_{k}}(t), x_{n_{k}}(t)-w(t)>d t .\right.
$$

Invoking Hypothesis (A) and Theorem 7 of [7], we obtain that

Hence,

$$
\begin{aligned}
& \int_{I} \underline{\lim }_{k \rightarrow \infty}<A\left(t, x_{n_{k}}(t)\right), x_{n_{k}}(t)-w(t)>d t \\
& \leq \varliminf_{k \rightarrow \infty} \int_{I}<A\left(t, x_{n_{k}}(t), x_{n_{k}}(t)-w(t)>d t .\right.
\end{aligned}
$$

$$
<\widehat{A} x, x-w>>\leq \varliminf_{k \rightarrow \infty}<<\widehat{A} x_{n_{k}}, x_{n_{k}}-w>>\text {. }
$$

Next we show that $\left.\widehat{A}\right|_{W_{p q}}$ satisfies the condition $(M)$. Let $x_{n} \longrightarrow^{w} x$ in $W_{p q}$, $\widehat{A} x_{n} \longrightarrow^{w} b$ in $V^{*}$ and $\left.\varlimsup_{n \rightarrow \infty}<<\widehat{A} x_{n}, x_{n}\right\rangle>\leq<<b, x>>$.

Due to (c)(i) and

$$
\begin{aligned}
& \varlimsup_{n \rightarrow \infty}<<\widehat{A} x_{n}, x_{n}-x>>\leq \varlimsup_{n \rightarrow \infty}<<\widehat{A} x_{n}, x_{n}>> \\
& -\lim _{n \rightarrow \infty}<<\hat{A} x_{n}, x>>\leq 0,
\end{aligned}
$$

there exists a subsequence of $\left(x_{n}\right)$, denoted by $\left(x_{n_{k}}\right)$, such that

$$
<\left\langle\widehat{A} x, x-w>>\leq \underline{\lim }_{k \rightarrow \infty}<<\widehat{A} x_{n_{k}}, x_{n_{k}}-w>>\right.
$$


for all $w \in V$. Thus,

$$
\begin{aligned}
& \leq \varlimsup_{k \rightarrow \infty}<<\widehat{A} x_{n_{k}}, x_{n_{k}}>>-\lim _{k \rightarrow \infty}<<\widehat{A} x_{n_{k}}, w>> \\
& \leq<<b, x-w>>,
\end{aligned}
$$

$$
\widehat{A} x=b .
$$

Similar to the proofs of Theorem 23.A and Lemma 2.5.1 of [4], one can verify that the following Lemmas.

Lemma 2. Under Hypothsis(F), $\widehat{f}: Z \rightarrow V^{*}$ is bounded and satisfies

(a) $\quad\|\widehat{f} x\|_{V^{*}} \leq \operatorname{const}\left(\left\|a_{2}\right\|_{q}+b_{2}\|x\|_{Z}^{p-1}\right)$;

(b) $\quad<<\widehat{f} x, x>>\leq\|\phi\|_{1}$, for all $x \in V$;

(c) $\hat{f}: Z \rightarrow V^{*}$ is continuous.

Lemma 3. If Hypotheses (A), $(\mathrm{F}),\left(H_{0}\right)$ are satisfied, and $x \in W_{p q}$ is a solution of (3.1), then there exist positive constants $M_{1}, M_{2}, M_{3}$, such that

$$
\begin{aligned}
& \max _{t \in I}|x(t)| \leq M_{1} ; \\
& \|x\|_{W_{p q}} \leq M_{2} ; \\
& \|\widehat{A} x\|_{V^{*}} \leq M_{3} .
\end{aligned}
$$

Note that (3.1) is equvalent to the following operator equation

$$
\left\{\begin{array}{l}
\dot{x}+\widehat{A} x=\widehat{f} x \\
x(0)=x_{0}
\end{array}\right.
$$

(See pp. 767-770, [3]).

Theorem 1. Suppose that Hypotheses of (A), $(\mathrm{F})$, and $\left(\mathrm{H}_{0}\right)$ hold, then the problem(3.1) admits a solution $x \in W_{p q} \subseteq C(I, H)$.

Proof. Let $\left\{w_{1}, w_{2}, \ldots, w_{n}, \ldots\right\}$ be a basis in X. We set $X_{n}=\operatorname{span}\left\{w_{1}, w_{2}, \ldots, w_{n}\right\}$, and introduce on the n-dimensional space $X_{n}$ the scalar product of the $H$-space $H$. Note that $X_{n} \subseteq X \subseteq H$.

Suppose that $x_{n_{0}} \longrightarrow^{s} x_{0}$ in $H$. The Galerkin equation of (3.1) can be constructed by

$$
\left\{\begin{array}{l}
\left.<\dot{x}_{n}(t), w_{j}>+<A\left(t, x_{n}(t)\right), w_{j}\right\rangle=<f\left(t, x_{n}(t)\right), w_{j}>, \quad \text { a.e.onI } \\
x_{n}(0)=x_{n_{0}}, \quad j=1,2, \ldots, n \\
w_{j} \in X_{n}, x_{n} \in X_{n}
\end{array}\right.
$$

By using Lemma 3, Hypotheses (A)(a)-(d), (F)(a)-(c), Proposition 27.7 of [3] and Carátheodory theorem ([7]), the Galerkin equation(3.3) must have a solution $x_{n}(\mathrm{n}=1,2, \ldots)$.

By Lemma 3, we know that there exists $M^{*}>0$ s.t.

$$
\max _{t \in I}\left|x_{n}(t)\right|+\left\|x_{n}\right\|_{W_{p q}}+\left\|\widehat{A} x_{n}\right\|_{V^{*}} \leq M^{*} .
$$

Hence, we can find a subsequence, again denoted by $\left\{x_{n}\right\}$, such that $x_{n} \longrightarrow^{w}$ $x$ in $W_{p q}, \widehat{A} x_{n} \longrightarrow^{w} b$ in $V^{*}$, and $x_{n}(T) \longrightarrow^{w} z$ in $H$. Using the same procedure of the proof of Lemma 30.4 of [3], we can verify that the limit elements $x, w$, and $z$ satisfy

$$
\left\{\begin{array}{l}
\dot{x}+b=\widehat{f} x \\
x(0)=x_{0}, \quad x(T)=z, \quad x \in W_{p q}
\end{array}\right.
$$

It follows from the Galerkin equation that

$$
<<\widehat{A} x_{n}, x_{n}>>=<<\widehat{f} x_{n}, x_{n}>>-\frac{1}{2}\left(\left|x_{n}(T)\right|^{2}-\left|x_{n 0}\right|^{2}\right) .
$$


Since $x_{n}(T) \longrightarrow^{w} x(T)$ in $\mathrm{H}$, we have

Hence

$$
|x(T)| \leq \varliminf_{n \rightarrow \infty}\left|x_{n}(T)\right| .
$$

Using Lemma 1, we assert that

$$
\varlimsup_{n \rightarrow \infty}<<\widehat{A} x_{n}, x_{n}>>\leq<<b, x>>.
$$

$$
\widehat{A} x=b \text {. }
$$

This shows that $x$ is a solution of (3.2), and hence a solution of (3.1).

\section{Existence of Admissible Trajectories and Optimal Controls}

We now consider the following controlled evolution system:

$\left\{\begin{array}{l}\dot{x}(t)+A(t, x(t))=f(t, x(t), u(t)), \quad \text { a.e.onI } \\ x(0)=x_{0}, \quad u \in \mathcal{U}_{a d}\end{array}\right.$

We impose some hypotheses.

.Hypothsis $\left(\mathbf{F}^{*}\right) . f: I \times Y \times E \rightarrow X^{*}$ satisfies:

(a) $\quad(t, x, u) \rightarrow f(t, x, u)$ is measurable;

(b) $\quad(x, u) \longrightarrow f(t, x, u)$ is continuous from $Y \times E_{w}$ into $X^{*}$,

here $E_{w}$ denotes the space $E$ with its weak topology;

(c) $\quad\|f(t, x, u)\|_{*} \leq a_{3}(t)+b_{3}\left(\|x\|_{Y}^{p-1}+\|u\|_{E}\right)$,

for all $x \in Y$ and $u \in E$, with $a_{3} \in L_{+}^{q}(0, T)$ and $b_{3}>0$;

(d)

$$
<f(t, x, u), x>\leq \widetilde{\phi}(t)+k\|u\|_{E}^{\frac{p}{p-1}},
$$

for all $x \in X$ and $u \in E$ with $\widetilde{\phi} \in L^{1}(0, T)$ and $k \in R$.

Hypothsis (U). $U: I \rightarrow P_{f}(E)$ is $L^{q}$-bounded multifunction.

By virtue of Theorem 1, we can conclude the existence of admissible trajectories of (4.1).

Theorem 2. Assume that Hypotheses $(A),\left(F^{*}\right),\left(H_{0}\right)$, and $(U)$ hold, then the problem (4.1) admits a solution corresponding to each date pair $\left(x_{0}, u\right) \in$ $H \times \mathcal{U}_{a d}$.

By Hypothesis $(\mathrm{U}), \mathcal{U}_{a d}$ is a bounded subset in $L^{q}(I, E)$. Set $P\left(x_{0}\right)=\left\{x_{u} \in\right.$ $W_{p q}, x_{u}$ is a solution of (4.1) corresponding to $u \in \mathcal{U}_{a d}$ and $\left.x_{0} \in H\right\}$, and $K(T)=\left\{y=x(T): x \in P\left(x_{0}\right)\right\}$. Utilizing the similar method of Lemma 3, we obtain

Lemma 5. Under Hypotheses of Theorem 2, there exists positive constants $M_{1}, M_{2}$, and $M_{3}$, such that for all $x \in P\left(x_{0}\right)$,

$$
\begin{gathered}
\max _{t \in I}|x(t)| \leq M_{1} ; \\
\|x\|_{W_{p q}} \leq M_{2} .
\end{gathered}
$$

Consider the optimal control problem (P):

$J(x, u)=\phi_{0}(x(T))+\int_{0}^{T} L(t, x(t), u(t)) d t \longrightarrow \inf$

$\left\{\begin{array}{l}\text { s.t. } \\ \dot{x}(t)+A(t, x(t))=f(t, x(t), u(t)), \quad \text { a.e.onI } \\ x(0)=x_{0}, \quad u \in \mathcal{U}_{a d}\end{array}\right.$

Suppose 
Hypothesis ( $\left.\mathbf{U}^{*}\right) . U: I \rightarrow P_{f c}(E)$ is $L^{q}$-bounded multi-function.

Hypothesis (F**). $f\left(., x_{n}(),. u_{n}().\right) \longrightarrow^{s} f(., x(),. u()$.$) in V^{*}$ as $x_{n} \longrightarrow^{s} x$ in $Z=L^{p}(I, Y)$ and $u_{n} \longrightarrow^{w} u$ in $L^{q}(I, E)$.

Hypothesis (L). $L: I \times X \times E \rightarrow R \cup\{+\infty\}$ s.t.

(a) $\quad(t, x, u) \rightarrow L(t, x, u)$ is measurable;

(b) $\quad(x, u) \rightarrow L(t, x, u)$ is l.s.c.;

(c) $\quad u \rightarrow L(t, x, u)$ is convex;

(d) $\quad \phi(t)-c\left(\|x\|+\|u\|_{E}\right) \leq L(t, x, u)$, a.e. on $I$,

with $\phi \in L^{1}(0, T)$ and $c \geq 0$.

Hypothesis (P). $P \equiv K(T) \cap Q \neq \phi$, where $Q$ denotes a weakly closed subset of $H$-space $H$.

Hypothesis $\left(\Phi_{0}\right) . \phi_{0}: P \subseteq H \rightarrow R$ is w.l.s.c.

Hypothesis $(\mathbf{J})$. There exists an admissible state-control pair $(x, u)$ such that

$J(x, u)<+\infty$.

Theorem 3. If Hypotheses $(A),\left(F^{*}\right),\left(F^{* *}\right),\left(H_{0}\right),\left(U^{*}\right),(L),(P),\left(\Phi_{0}\right)$, and $(J)$ hold, then the problem $(\mathrm{P})$ has an optimal pair $(x, u)$.

Proof Suppose that $\left(x_{n}, u_{n}\right)$ is the minimizing sequence, then it satisfies the following initial value problem:

$$
\left\{\begin{array}{l}
\dot{x}_{n}(t)+A\left(t, x_{n}(t)\right)=f\left(t, x_{n}(t), u_{n}(t)\right) \\
x_{n}(0)=x_{0}
\end{array}\right.
$$

Recalling Lemma 5 and boundedness of $\mathcal{U}_{a d}$ in $L^{q}(I, E)$, by passing to a subsequence, if necessary, we may assume that $x_{n} \longrightarrow^{w} x$ in $W_{p q}, \widehat{A} x_{n} \longrightarrow^{w} b$ in $V^{*}, x_{n}(T) \longrightarrow^{w} z \in Q$ in $H$, and $u_{n} \longrightarrow^{w} u$ in $L^{q}(I, E)$. By virtue of Mazur Lemma and Hypothesis $\left(\mathrm{U}^{*}\right)$, we have

$$
u(t) \in \overline{c o} w-\lim _{n} \sup \left\{u_{n}(t)\right\}_{n \geq 1} \subset U(t) \text {, a.e. on } I .
$$

Hence, $u \in \mathcal{U}_{a d}$. As in the proof of Theorem 1, we can prove that $x$ satisfies

$$
\left\{\begin{array}{l}
\dot{x}+\widehat{A} x=f(., x(.), u(.)) \\
x(0)=x_{0} \\
x(T)=z \in Q
\end{array}\right.
$$

Therefore, $x(T) \in P \subseteq H$. Next, it follows from (4.2) that $<<\dot{x}_{n}-\dot{x}, x_{n}-$ $x>>+<<\widehat{A} x_{n}-\widehat{A} x, x_{n}-x>>=<<f\left(., x_{n}(),. u_{n}().\right)-f(., x(),. u()),. x_{n}()-$. $x()>.>$.

By Lemma 1, we obtain that

$c\left\|x_{n}-x\right\|_{V}^{p} \leq d\left\|x_{n}-x\right\|_{\mathcal{H}}^{2}$

$+<<f\left(., x_{n}(),. u_{n}().\right)-f(., x(),. u()),. x_{n}()-.x()>.>$.

Therefore, by Hypothesis $\left(\mathrm{F}^{* *}\right), W_{p q} \hookrightarrow L^{p}(I, Y)$ compactly, we claim that $x_{n} \longrightarrow^{s} x$ in $V$. Since $x_{n}(T) \longrightarrow^{w} x(T)$ in $H$, Hypothesis $\left(\Phi_{0}\right)$ infers that

$\phi_{0}(x(T)) \leq \varliminf_{n \rightarrow \infty} \phi_{0}\left(x_{n}(T)\right)$.

By applying Hypothesis (L) and Balder theorem 2.1 ([6]), we conclude that $\int_{0}^{T} L(t, x(t), u(t)) d t \leq \underline{\lim }_{n \rightarrow \infty} \int_{0}^{T} L\left(t, x_{n}(t), u_{n}(t)\right) d t$

Consequently, 
$J(x, u) \leq \underline{\lim }_{n \rightarrow \infty} J\left(x_{n}, u_{n}\right)=\lim _{n \rightarrow \infty} J\left(x_{n}, u_{n}\right)$.

This shows that $(x, u)$ is the desired optimal pair.

\section{Examples}

Our results can be used to the following the initial boundary value problem of parabolic system of order $2 \mathrm{~m}$

$\left\{\begin{array}{lc}\frac{\partial}{\partial t} x(t, z)+L(t) x(t, z)=f(t, z, x(t, z)), & \text { on } I \times G \\ D^{\beta} x=0 & \text { on } \times \partial G, \quad|\beta| \leq m-1 \\ x(0, z)=x_{0}(z), & \text { on } G\end{array}\right.$

where $L(t) x(t, z)=\sum_{|\beta| \leq m}(-1)^{|\beta|} D^{\beta} A_{\beta}(t, z, \theta(x(t, z)))$,

$f(t, z, x(t, z))=\sum_{|\beta| \leq k}(-1)^{|\beta|} D^{\beta} f_{\alpha}(t, z, \eta(x(t, z))), \beta=\left(\beta_{1}, \beta_{2}, \ldots, \beta_{n}\right)$ is n-tuple of nonnegative integers, $|\beta|=\sum_{i=1}^{m} \beta_{i}, D^{\beta}=D_{1}^{\beta_{1}} D_{2}^{\beta_{2}} \ldots D_{n}^{\beta_{n}}, D_{i}=\frac{\partial}{\partial x_{i}}$, $\theta(x)=\left\{D^{\beta} x:|\beta| \leq m\right\}, \eta(x)=\left\{D^{\beta} x,|\beta| \leq k\right\}$, and $k<m$. Take

$X=W_{0}^{m, p}(G), \bar{Y}=W_{0}^{k, p}(G), H=L^{2}(G)$.

Their dual spaces are

$X^{*}=W^{-m, q}(G), Y=W^{-k, q}(G), H^{*}=H$,

respectively. From Soblev embedding theorem, we know that

$$
X \hookrightarrow Y \hookrightarrow H \hookrightarrow Y^{*} \hookrightarrow X^{*},
$$

with all embedings being continuous, dense, and compact([1]). Define,

$a_{1}(t, x, y)=\int_{G} \sum_{|\beta|<m} \xi_{\beta}(t, z, \theta(x)) D^{\beta} y d z$

$a_{2}(t, x, y)=\int_{G} \sum_{|\beta|=m} \xi_{\beta}(t, z, \theta(x)) D^{\beta} y d z$, for all $x, y \in X$,

$b(t, x, y)=\int_{G} \sum_{|\beta| \leq k} f_{\beta}(t, z, \eta(x)) D^{\beta} y d z$.

Under some reasonable assumptions similar to Section 30.4 of [3], one can verify that there exist operaters $A_{i}(.,):. I \times X \rightarrow X^{*}(i=1,2)$ and $f(.,$.$) :$ $I \times Y \rightarrow X^{*}$ satisfying

$<A_{i}(t, x), y>=a_{i}(t, x, y), i=1,2$;

$<f(t, x), y>=b(t, x, y)$.

Define

$A(t, x)=A_{1}(t, x)+A_{2}(t, x)$.

By Proposition 26.16 of [3] and Corrollary 26.14 of [3], we can check that the operaters $A$ and $f$ satisfy the assumptions of Theorem 1 . Hence, the existence of weak solutions can be proved. Futher, under some assumptions of Example 1 of [1], we can solve the following optimal control problem:

$$
\begin{aligned}
& J(x, u)=\int_{G}|x(T, z)| d z+\int_{0}^{T} \int_{G} \frac{1}{2}\left|x(t, z)-y_{0}(z)\right|^{2} d t d z \\
& \quad+\frac{\theta}{2} \int_{0}^{T} \int_{G}|u(t, z)|^{2} d t d z \longrightarrow \text { inf, } \\
& \text { s.t. } \begin{array}{lc}
\frac{\partial}{\partial t} x(t, z)+L(t) x(t, z)=f(t, z, x(t, z))+b(t) u(t, z), & \text { on } \times Q \\
D^{\beta} x=0, & \text { onI } \times \partial G, \quad|\beta| \leq m-1 \\
x(0, z)=x_{0}(z) & \text { on } G
\end{array}
\end{aligned}
$$




\section{References}

[1] N.S.Papgeorgiou, Optimal control of nonlinear evolution equations with nonmonotone nonlinearities, Journal of optimization theory and application, Vol. 77, No. 3, pp.643-660, 1993.

[2] N.S.Papageorgiou. Properties of the relaxd trajectories of evolution equations and optimal control. SIAM Journal on control and optimization, Vol. 27, No.2, pp.267-288, 1989.

[3] E.Zeidler, Nonlinear Functional Analysis and its Applications II, SpringerVerlag, New York, Berlin Heidelberg London Paris,Tokyo, 1992.

[4] N.U.Ahmed and K.L.Teo, Optimal control of distributed parameter systems. North-Holland, New

York, Oxford, pp.368-391, 1981.

[5] N.U. Ahmed \& X.L. Xiang, Nonlinear Uncertain Systems and Necessary Conditions of Optimality, SIAM Journal on control and optimization, Vol. 35, No. 5, Sept. 1997.

[6] E.J.Balder, Necessary and sufficient conditions for $\mathrm{L}^{1}$-strong-weak lower semicontinuity of integral functions, nonlinear analysis, Vol.11, No. 12, pp.1399-1403, 1987.

[7] R.F.Curtain, Functional analysis in modern applied mathematics, United States edition published by academic press inc. 111 Fifth Avenue, New York, New York 100003, 1977.

[8] Xia Daoxing, Real varation function and functional analysis, published by Education House, Book 2, 1987. 
Part II. Stochastic Systems 